\title{
Status and developments in EOSTAR, a model to predict IR sensor performance in the marine environment
}

\author{
Gerard J. Kunz $^{\mathrm{a}^{*}}$, Marianne A.C. Degache ${ }^{\mathrm{a}}$, Marcel M. Moerman ${ }^{\mathrm{a}}$, \\ Alexander M.J. van Eijk ${ }^{a}$, Filip P. Neele ${ }^{a}$, \\ Stephen M. Doss-Hammel ${ }^{\mathrm{b}}$ and Dimitri Tsintikidis ${ }^{\mathrm{b}}$ \\ ${ }^{a}$ TNO Physics and Electronics Laboratory, Department of Electro-Optic \\ The Hague, The Netherlands, \\ ${ }^{\mathrm{b}}$ Atmospheric Propagation Branch, Code 2858, SPAWAR Systems Center \\ San Diego, CA 92152, USA
}

\begin{abstract}
The application of long-range infrared observation systems is challenging, especially with the currently available high spatial resolution infrared camera systems with resolutions comparable with their visual counterparts. As a result of these developments, the obtained infrared images are no longer limited by the quality of system but by atmospheric effects instead. For instance, atmospheric transmission losses and path radiance reduce the contrast of objects in the background and optical turbulence limits the spatial resolution in the images. Furthermore, severe image distortion can occur due to atmospheric refraction, which limits the detection and identification of objects at larger range. EOSTAR is a computer program under development to estimate these atmospheric effects using standard meteorological parameters and the properties of the sensor. Tools are provided to design targets and to calculate their infrared signature as a function of range, aspect angle, and weather condition. Possible applications of EOSTAR include mission planning, sensor evaluation and selection, and education. The user interface of EOSTAR is fully mouse-controlled, and the code runs on a standard Windows-based PC. Many features of EOSTAR execute almost instantaneous, which results in a user friendly code. Its modular setup allows its configuration to specific user needs and provides a flexible output structure.
\end{abstract}

Keywords: EOSTAR, infrared propagation, electro-optical, atmospheric turbulence, atmospheric refraction

\section{INTRODUCTION}

The technological developments of electro-optical sensors, such as visual and infrared (IR) sensor systems, have allowed for an increased use of these systems in long-range surveillance applications. Modern IR sensors can reliably detect temperature differences of 0.1 degree and have a high spatial resolution, which in principle permits the detection and identification of distant targets against their background. The passive nature of the sensors and the high spatial resolution provide competitive advantages over radar in specific applications. However, the sensor performance that can be anticipated on the basis of the technical specifications is often limited by atmospheric effects, a situation that is not always recognized by designers and users.

The atmosphere reduces the radiation received from a target (transmission losses), blurs the image due to turbulence and distorts the image due to refraction, effects that are strongly related to the prevailing meteorological condition. Several models have been reported for the prediction of transmission losses ${ }^{1-4}$, refraction ${ }^{5-12}$ and turbulence ${ }^{13-15}$, but application of these models is laborious and complex and the individual model results are difficult to combine. Although a few other (complete signature) models are known from literature ${ }^{16-21}$ these are either not running under Windows on standard PC's, do not account for atmospheric refraction and turbulence or do not generate synthetic images.

\footnotetext{
* Contact information: Gerard.Kunz@fel.tno.nl, phone: +31 70374 0460, TNO Physics and Electronics Laboratory, P.O. Box 96864 , 2509 JG The Hague, The Netherlands; http://www.tno.nl
} 
A need exists for a user-friendly code that (a) provides the coupling between outputs of various models and (b) reconciles the discrepancies between various data formats and requirements. The EOSTAR model suite ${ }^{22-25}$ is currently being developed to fill this gap. While the underlying models are identical (e.g., bulk models, ray tracing model, ANAM, MODTRAN), two separate user interfaces have been developed for two main applications of the modeling approach. First, the ARTEAM module provides databases of sensors, targets and environmental scenarios allowing quick selection of model input. Its main output consists of coverage diagrams of detection range, which makes the module suited for mission planning purposes. Second, the EOSTAR module allows for the detailed specification of input parameters. While one of its main outputs consists of the synthetic sensor image, the interface also provides detailed intermediate results and properties of scientific interest. This, in addition, makes EOSTAR an excellent educational tool for studying the impact of various atmospheric processes on the image quality.

This contribution focuses on the EOSTAR module. The program is a mouse-driven, Windows-based container for modules that calculate various atmospheric effects for a given infrared sensor system. Currently focusing on applications in the marine surface layer, EOSTAR is, among other parameters, driven by standard meteorological data as air and surface temperature, relative humidity, wind speed and air pressure in combination with the sensor specifications. Based on these input data, micrometeorological models are solved to provide profiles of optical refractivity and turbulence. EOSTAR then uses these results to predict image distortion, scintillation, blur and image dancing. In addition, the spectrally resolved background radiance and transmission losses due to aerosols and molecules are calculated. Pathintegrated transmission and path radiance are computed for a particular target in the scene. EOSTAR is also endowed with modules to work with user-defined targets, constructed from thousands of elementary segments each with their own physical and optical properties. Using an extended input set of meteorological parameters, the spectral emission of each segment is calculated. Alternatively, users can read their own spectra from files, which may be useful when dealing with exhaust plumes or other sensitive information. False color images of, e.g., the spectrally integrated radiance or the apparent temperature, can be produced taking into account atmospheric refraction, transmission, path radiance, and turbulence. These synthetic images represent the sensor image of the scene under the specified conditions. Final and intermediate results can be exported as hard copy or in electronic format for further post-processing in specific user applications.

This paper discusses and presents the current status and recent developments of EOSTAR illustrated with some examples demonstrating its capabilities. The paper starts with a short introduction on the micrometeorological models, which are used to estimate the vertical profiles of temperature, humidity, pressure and wind speed, and the optical properties of the atmosphere. The next section discusses the ray tracer that predicts the observation paths of the individual sensor pixels (ray trajectories), which are used to calculate image distortion due to refraction and turbulence. Finally, the capabilities of a new target input module that gives users full control over the target geometry and, its radiative properties are discussed. Different examples throughout the paper illustrate the capabilities of the EOSTAR model.

\section{MICROMETEOROLOGY}

The current version of EOSTAR is developed for long-range observations in the lowest tens of meters in the marine atmosphere, commonly known as the marine surface layer. For most applications of EOSTAR, it is assumed that this layer is horizontally homogeneous, but the program can be extended to work with different horizontal segments, each with its own meteorology. The marine surface layer is considered as a constant flux layer in which mixing takes places through convection and wind activity ${ }^{26-29}$. The set of non linear equations describing wind stress, heat and water vapor fluxes in the surface layer have been known for a long time and several models have been proposed to solve this set of equations involved for a given set of input values of surface temperature, air temperature, humidity, pressure and wind speed at a certain height. Different methods to solve the set of equations can be found in the literature. These micrometeorological or bulk models yield the scaling parameters and roughness lengths for temperature, humidity and wind speed, respectively $t^{*}, q^{*}$ and $u^{*}$, and $z_{\mathrm{ot}}, z_{\mathrm{oq}}$ and $z_{\mathrm{om}}$, and the Monin-Obukhov length $L$. These parameters, in combination with the stability correction functions $\Psi(z / L)$, allow the calculation of the vertical profiles of temperature, humidity and wind speed. Within EOSTAR, 6 different micrometeorological models ${ }^{30-34}$ are solved instantly and simultaneously. Although all results can be presented in a table format for direct comparison, only one set is used for further processing. 
Figure 1 shows a number of features of the bulk parameterization module in EOSTAR. Input data can be entered graphically (left-hand panel) or numerically (middle panel), or can be loaded from ASCII files. Data files can be processed sequentially, either automatically ("movie") or with the user stepping manually through the various cases. In addition, provisions can be made to couple the input module to a meteorological station to obtain a continuous update of the micrometeorological data and profiles of temperature, humidity and wind speed in the marine surface layer.
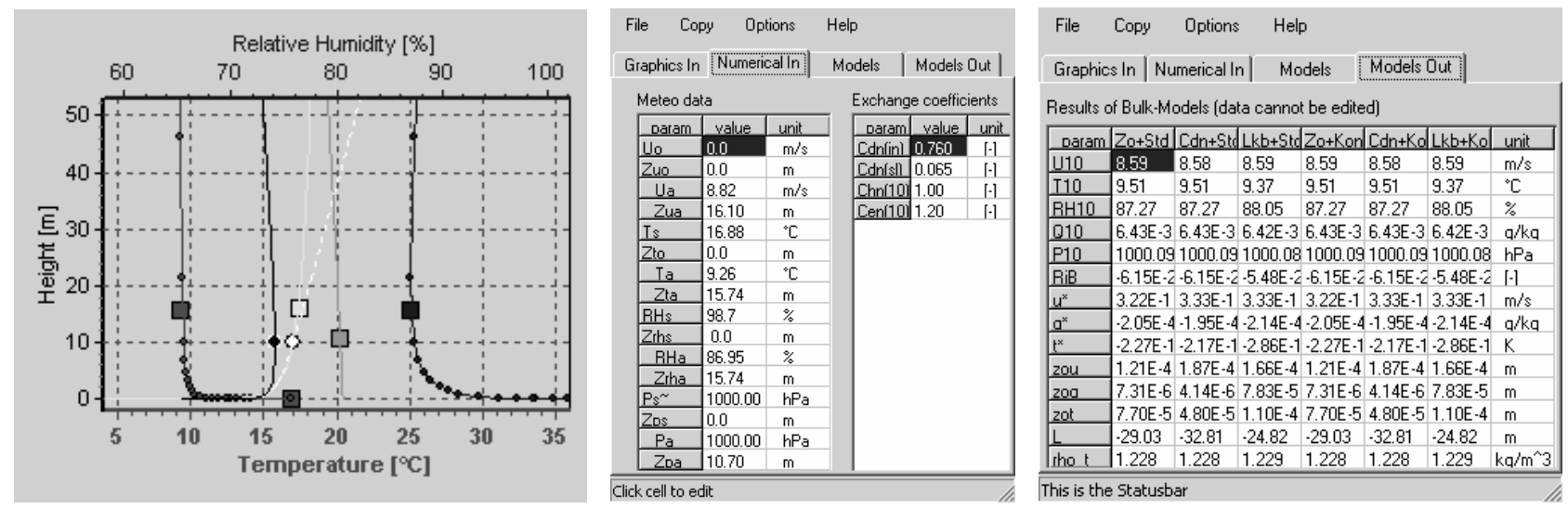

Figure 1: Bulk meteorological module in EOSTAR with the graphical input module (left), the numerical input module (middle) and the numerical output module (right panel) showing the results of 6 different models.

The bulk meteorological module yields (modified) logarithmic vertical profiles only. In cases that the atmospheric vertical structure is not well described by the bulk parameterization theory, or when additional meteorological data (e.g., height profiles from buoys) are available, EOSTAR offers a tool to specify (non-logarithmic) user-defined meteorological profiles. These profiles are subsequently used to evaluate the refractive effects, calculate transmission, path radiance, scintillation, etc.

\section{OPTICAL REFRACTION AND TURBULENCE}

The refractive index of air is not constant but varies with temperature, humidity, pressure and wavelength. These refractive index variations play a crucial role in atmospheric refraction and turbulence effects. The variation with height, $\partial n / \partial z$, especially near the surface where the gradient is strong, is responsible for the deformation of images, an effect that on land is often observed over sun-heated roads. Similar effects occur over the sea while observing targets at large distances. Variations of the refractive index with temperature, $\partial n / \partial T$, and humidity, $\partial n / \partial Q$, are responsible for turbulent effects (variations with pressure are negligible). These effects are quantified in the local value of the refractive index structure parameter, $C_{\mathrm{n}}^{2}$.

Several optical application models describing the refractive index of air for optical application can be found in the literature $^{11,35}$, but only a few are independent ${ }^{9,10,12,36}$ and these provide comparable results for the spectral interval of interest. Within EOSTAR, the extended functions proposed by Edlén ${ }^{9,10}$ are used because they are defined over a wide spectral range. An analysis of the results obtained with this model indicates that the difference between the visible and infrared refractivity, $N_{v i s}-N_{I R}$ with $N=(n-1) \cdot 10^{6}$, is about 5 units, but that the variability of the refractive index with temperature and humidity is practically negligible. The latter is a valuable result, indicating that image distortion due to atmospheric refraction, which is driven by $\partial n / \partial z$, thus by $\partial n / \partial T$ and $\partial n / \partial Q$, is practically independent of wavelength $^{37}$, making, consequently, $C_{\mathrm{n}}{ }^{2}$ also almost independent of the above quantities. On the other hand, effects of scintillation and blur are wavelength dependent, since the equations describing these phenomena do not only include $C_{\mathrm{n}}{ }^{2}$, but also the wavelength. 
EOSTAR calculates the vertical profile of the refractive index of air using the vertical profiles of temperature, humidity and pressure as illustrated in section 2 . This refractivity profile is used to calculate the paths of the individual sensor pixels (ray tracing, see section 4.1) and to calculate the distorted synthetic camera image (section 5). Scintillation, blur, and image dancing are based on path integrated, weighted values of the refractive index structure parameter $C_{\mathrm{n}}^{2}$, which, as shown above, depends on air temperature and humidity, the scaling parameters $t^{*}, q^{*}, u^{*}$, the Monin-Obukhov length $L$ and several other functions available in the literature ${ }^{30,38-46}$. Profiles of the refractivity $(N)$ and modified refractivity $\left(M=N+0.157 *_{z}\right)$ are displayed in the meteorological module (see the black and the white curves in Figure 1, left panel, respectively). The profile of $C_{\mathrm{n}}{ }^{2}$ is instantaneously available after updating the meteorological data or changing the spectral band. Scintillation, blur and image motion are calculated on the basis of the vertical profile of $C_{\mathrm{n}}{ }^{2}$ and the ray paths (see next section), and are also updated instantaneously. All results can be copied to ASCII files, clipboard or printer.

\section{RAY TRAJECTORIES AND ANALYSIS}

\subsection{Ray tracer description}

The 'sensor ray trajectories' are the basis for evaluating the effects of atmospheric refraction and path-integrated effects (e.g., transmission, radiance, blur, and scintillation) on the image. The ray trajectories represent the observation paths of the individual sensor pixels. In a homogeneous medium with a constant refractive index, these ray trajectories will be straight lines. However, this is never the case in the atmosphere where the vertical profiles of pressure, temperature and humidity induce variations in the vertical profile of refractive index. Even with constant temperature and humidity, the pressure gradient alone induces some super-refraction (optical horizon beyond the geometrical horizon).

Ray trajectories cannot simply be described with standard functions but must be constructed successively 'as the ray propagates' in the atmosphere, using the local ray direction and local refractive index gradient. For long-range imaging, the Earth curvature must also be taken into account and the locally assumed horizontal stratification of the refractive index must be considered as spherically stratified. The accuracy of the trajectories depends on the step size in the propagation direction and the sample interval of the refractive index vertical profile. Two different approaches have been reported $^{5,7,8,47,48}$. The first one is based on Snell's law under the assumption of a constant refractive index within the layers. This leads to straight rays within the layers, which bend or reflect on the boundaries of the layers. The second method is based on the assumption of a constant gradient of the refractive index within the layers, which results in parabolic ray segments within the layers and discontinuities on the boundaries. Both methods yield discontinuities in the trajectories on the boundaries of the layers, which can be visualized in EOSTAR by zooming in on the data. Both approaches give comparable results but the efficiency of the latter is somewhat higher and therefore applied in EOSTAR.

EOSTAR allows the selection of the number of rays over the field of view of the sensor and the horizontal increment along the trajectory. By default 50 rays are taken with a horizontal step size of $50 \mathrm{~m}$ resulting in a response time of a few tenths of a second for a complete set of ray trajectories. Trajectories are displayed within a box of user-selected height and range, while taking into account the height of wind-driven surface waves. A switch is available to bypass the wave module and to work with a user-defined wave height. The observation directions and the ranges of both the geometrical horizon and the optical horizon are also calculated within the ray tracing module. The first is simply calculated from the height of the sensor and the radius of the Earth. The optical horizon, on the other hand, is defined as the lowest ray skimming over the sea towards the sky and is calculated with an accuracy of $10 \mu \mathrm{rad}$. This definition implies that the optical horizon is not just a function of the sensor height, but also a function of the prevailing atmospheric conditions that affects the refractive index. In the case of super-refraction or a positive ASTD (air-sea temperature difference), thus under stable conditions, the optical horizon is always at larger range than the geometrical horizon. In the case of subrefraction or negative ASTD, thus under unstable conditions, the horizon relative position depends on the height of the sensor above the surface. For low-placed sensors, the optical horizon is at a larger range than the geometrical horizon whereas the opposite holds for the case of high-placed sensors. Hence, it is not straightforward to determine the atmospheric stability status from a measurement of the relative ranges or positions of optical and geometrical horizons.

Each ray trajectory can be considered as the axis of the instantaneous field of view (IFOV) of a single detector element. The curve is launched from the centre of this pixel, crosses the middle of the optics and starts its propagation path in the 
atmosphere at this height and at an elevation angle corresponding to the particular detector element. A fan of camera ray trajectories is obtained by considering several detector elements on a vertical array. However, this is a first order approach, which neither accounts for the size of the detector element nor for the aperture of the optics. This approach has been refined by deJong ${ }^{49}$, who also suggested other improvements for the construction of the ray trajectories.

EOSTAR visualizes the ray trajectories in a rectangular coordinate system with the horizontal distance over the Earth surface being the horizontal axis and the height above the surface being the vertical axis (see left-hand side panel in Figure 2). The sensor is represented by the square at zero range at a height of $18 \mathrm{~m}$ above the surface. Visualization and interpretation of the data in this way is much easier than in a chart with a curved Earth surface, although it might be confusing at first glance that the straight line from the sensor towards the geometric horizon becomes curved (see the right most curve in the left-hand side panel of Figure 2).
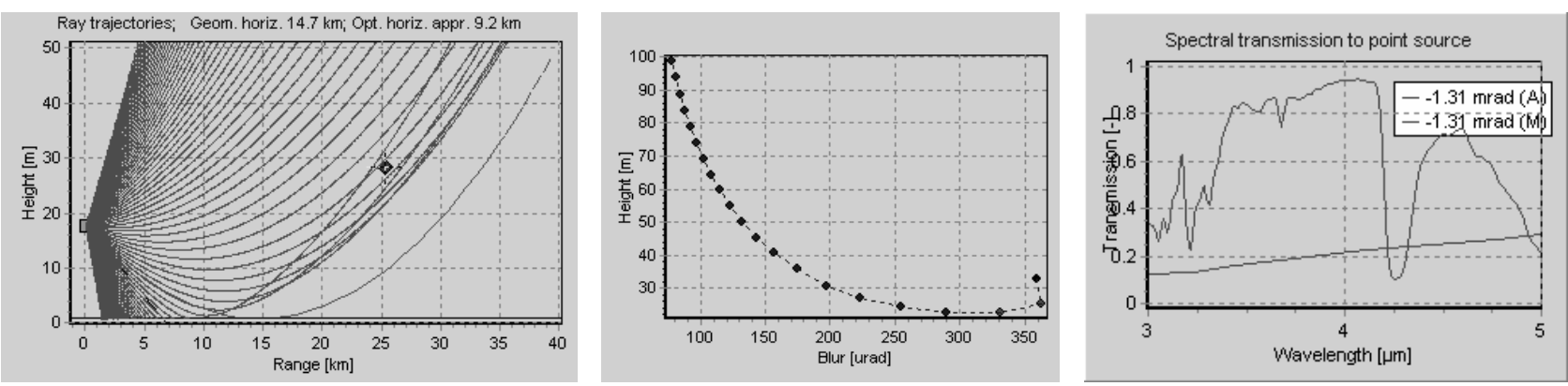

Figure 2: Example of a set of ray trajectories with a mouse controlled cursor (left panel). The middle panel shows the atmospheric vertical blur profile along the paths between the camera (indicated by the square at zero range in the left panel) and the position of the cursor (dot). The right panel shows the spectra of molecular and aerosol transmission for the path between the camera and the cursor.

Once the ray trajectories have been calculated, the user has full access to local and path-integrated parameters. A few of those are presented in the middle and right-hand side panels of Figure 2. The solid dot in the left-hand panel (at a range of approximately $23 \mathrm{~km}$ ) is a mouse-controlled cursor that can be dragged and dropped to simulate the position of a distant point source. Subsequently, the program calculates and returns instantaneously the parameters of interest, e.g., meteorological data at this position, direction of observation and ray number, transversal coherence length and the spectrally resolved transmission, scintillation, and blur along the selected path.

\subsection{Tools for analysis}

Vertical transfer functions describe the relationships between ray properties (e.g., angle of departure, height above the surface or local angle of the ray) in a refracting and a non-refracting atmosphere at the range of interest. EOSTAR provides several vertical transfer functions to study propagation effects in more detail. One example, given in the left panel of Figure 3, is the functional relation between the actual or geometric (non-refracted) and the apparent (refracted) observation direction of a point source at a given distance. Thus, the transfer function coincides with the line $y=x$ in a non-refracting atmosphere. This transfer function gives insight into the atmospheric refraction in the vertical plane at the range of the cursor ${ }^{24,50,51}$. This is illustrated by the vertical bar in the figure representing the position of a point target that would be visible at an elevation of $-1.5 \mathrm{mrad}$ in a non-refracting atmosphere. There are two crossings with the transfer function, indicating that this point source will be seen in the refracting atmosphere at two elevations $(-2.3 \mathrm{and}-1.2 \mathrm{mrad})$, and, hence, a mirage condition (two images of a single target). In a similar manner, it can be demonstrated that a point target, visible at $-2.5 \mathrm{mrad}$ in a non-refracting atmosphere, will not be visible at all in the refracting atmosphere. The horizontal lines in the figure provide information about the horizons: the solid green line indicates the direction of the optical horizon and the broken red line refers to the geometric horizon.

The vertical transfer function is a valuable tool for predicting the occurrence of mirages. This occurrence is based on the existence of negative ASTD, but this is only correct to first order. The occurrence of mirages depends on the structure of the refractive index vertical profile. In addition to ASTD, the refractive index profile depends also on humidity and, above a sea surface, wind speed. The latter parameter represents the effects of wind-induced waves that limit in effect the minimum ray height above the surface and, therefore, the occurrence of mirages at elevations closest to the horizon. 

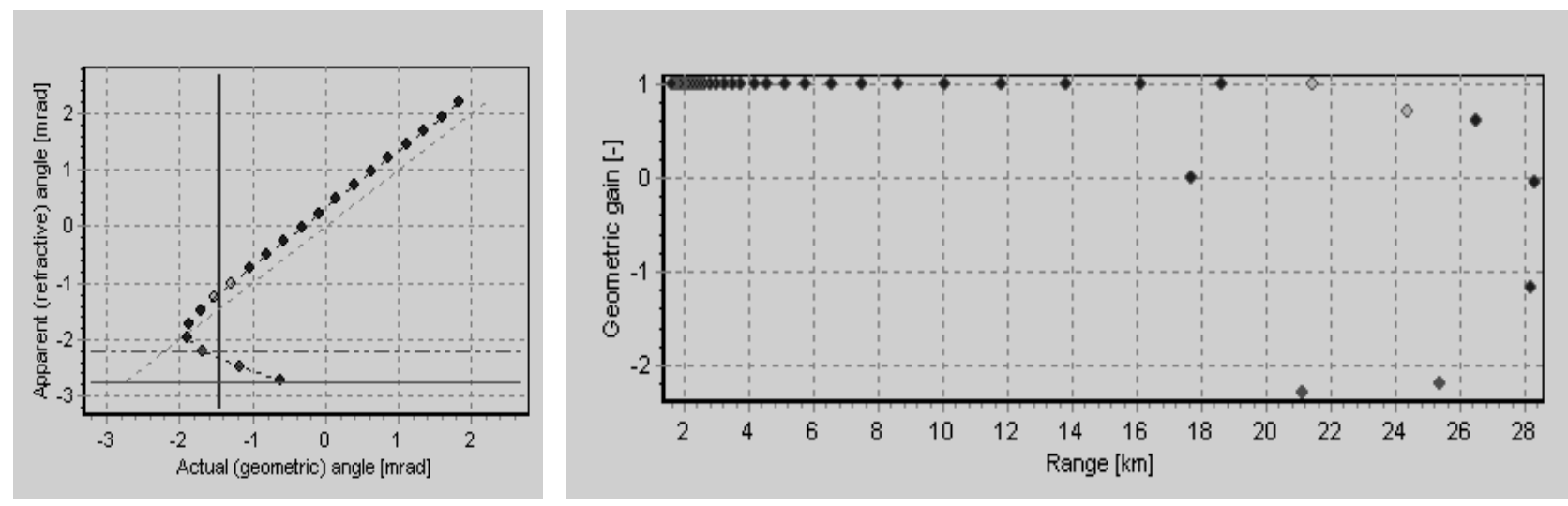

Figure 3: Left panel: example of a vertical transfer function during a mirage condition, describing the atmospheric distortion over a distance of $23 \mathrm{~km}$. The solid dots show the relation between the refracted ray directions (represented by the vertical axis) and the geometric ray directions (represented by the horizontal axis). The solid brown vertical line represents the geometrical observation direction of the cursor in the ray trajectory chart. The solid green horizontal line is the direction of the optical horizon and the broken red horizontal line refers to the geometric horizon. The right panel shows the refractive propagation factor as a function range at a height of $25 \mathrm{~m}$.

If vertical transfer functions provide information about parameters as functions of height at fixed range, horizontal transfer functions assess parameters as functions of range at a fixed height. This may provide useful information when studying, for example, scintillation or blur of an incoming point target. EOSTAR includes various horizontal transfer functions, one of which is shown in the right-hand panel of Figure 3. This particular function, known as the refractive propagation factor $F^{2}$ (also referred to as magnification or geometric gain), provides the ratio of a pixel's cross-section in a refracting and a non-refracting atmosphere as a function of range ${ }^{16,52}$. The propagation factor changes the size of the imaged target but does not affect its irradiance in the camera focal plane. As a result, the signal strength corresponding to each of the individual ray trajectories (for targets larger than a single pixel) is invariant under atmospheric refraction. However, the total power received from a target, including target images that fall within a single pixel, will be affected by the propagation factor.

\subsection{Passive ranging}

As mentioned above, the bundle of ray trajectories roughly describes the observation cones of individual sensor pixels in the refracting atmosphere. The cross-section of such a cone depends on the instantaneous field of view (IFOV) of a pixel, the distance from the sensor and the atmospheric refraction. A point source strictly moving inside the volume of such a cone remains at a fixed position within the image. Consequently, there is no information on the distance of the target in the image. However, if an additional image is taken from a different height and the same point source is imaged from the new height, then parts of the two cones overlap resulting thus in a well-defined range and height interval. Thus, images of targets taken at two different heights contain information on the position of the target (vertical stereoscopic viewing), even in the presence of refraction.

A recent study ${ }^{51}$ suggests that it is possible to retrieve the range of a target when observed under mirage conditions. This technique is known as passive ranging and could be very useful in applications when the observer does not want to reveal his presence. EOSTAR is currently being modified to include a passive ranging tool.

Since the refractive index is a function of wavelength, one might be tempted to assume that images of the same target taken in different spectral bands are refracted differently and thus provide information on the target position. This is true in principle, but, it is not the refractive index itself, but its vertical gradient, and thus the vertical variations of temperature, humidity and pressure, that determine the refraction-induced image distortions. As discussed in section 3 , the wavelength dependence of the vertical gradient of refractive index is negligible over the electro-optical wavelength domain, which renders passive ranging from hyper-spectral imaging impractical. 


\section{SYNTHETIC SENSOR IMAGES}

The earlier versions of EOSTAR contained two image modules. The first was a 'target input module' used to select a pre-defined target in a non-refracting and non-turbulent atmosphere. The second was a 'target output module' presenting the image of the selected target as observed through a refracting and turbulent atmosphere. These modules only allowed studying geometric effects, i.e., the effect of the refracting atmosphere on the target shape. These two modules are delivered with the free EOSTAR demo version on the TNO-FEL website http://www.tno.nl/instit/fel/os/fac/eostar.html.

Presently, efforts are underway to significantly upgrade the target modules in EOSTAR. These improvements are driven by the high interest in the synthetic sensor images of realistic targets in a refractive atmosphere. It is now possible to import externally defined targets into EOSTAR, e.g., coming from a CAD application, with the ability to assign physical and optical properties to each target segment. These data, in conjunction with the selected atmospheric condition and a suitable target signature model, allow for the calculation of the stationary skin temperature, spectral emission and apparent temperature for each of the segments. The signature module requires special preparations and accounts for the incoming radiance from the sun, sky, and the sea surface, the direction of the sun, wind speed and the orientation of the target segment. The signature module can be by-passed by reading radiance spectra of segments from an external data file, e.g., for exhaust plumes.

The input module has been reconstructed so that multiple targets can be placed in the scene, each with its own properties, position and direction. Using the mouse, the user can position and orient each target, and retrieve instantaneously the properties of each single segment of a target. Also the position and direction of the camera, including the focal length, can be adjusted with simple mouse actions. Scenes are presented as false color images (or gray tone), representing the various available parameters, e.g., the skin temperature, band integrated radiance or apparent temperature. The scene can be viewed from any desired aspect angle also by simple mouse actions. Information on the total projected area, radiance and apparent temperature are updated instantaneously and projected in the bottom of the images. An example of an IR image of a ship as seen from sun and shadow sides is shown in Figure 4.
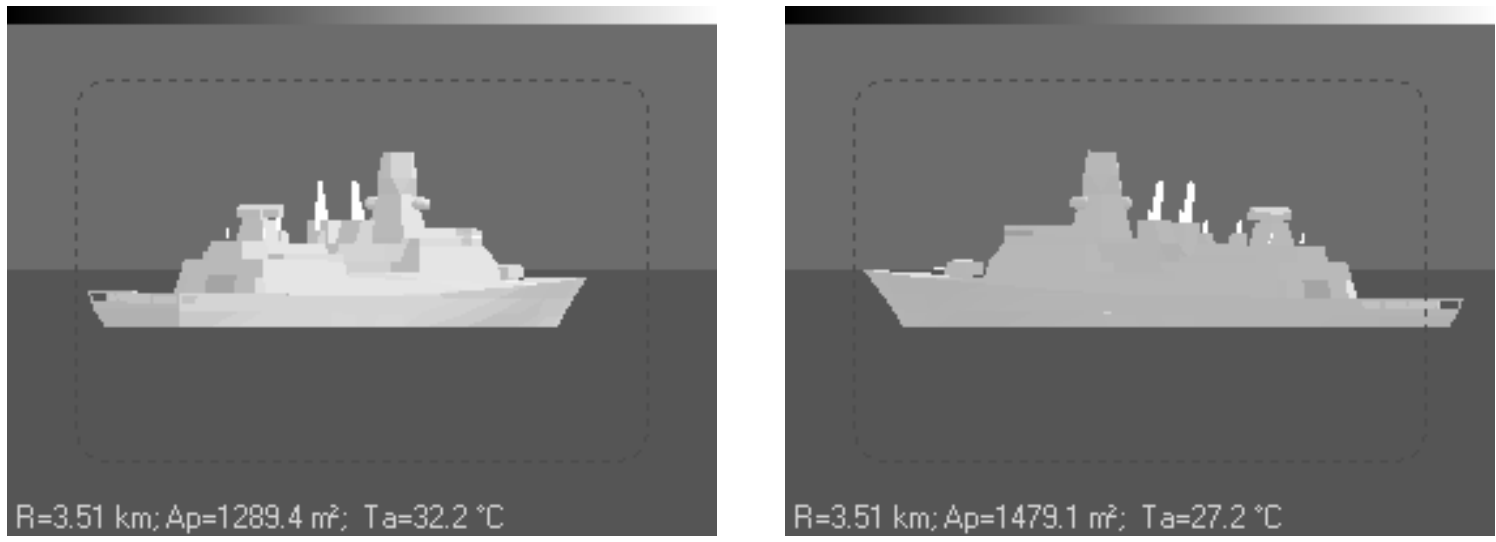

Figure 4: False color representation of a simulated infrared image of a ship (scale in arbitrary units) as seen from two sides. No refraction, blur or noise is added to these images. (Projected data in the bottom is not representative for the presented synthetic target).

The target output module that visualizes atmospheric effects on the target image has also been redesigned. This module now takes the input image (as shown in Figure 4) and multiplies the emission spectra of the segments with the transmission spectra along the ray trajectories and adds the path radiance spectra. Subsequently, the resulting image is refracted and blurred using the previously discussed modules of EOSTAR. Next, noise (as specified in the sensor properties module) can be added to the image. Finally, the resulting image can be re-sampled simulating the spatial resolution of the detector array. Examples of output images in different modes of the program are presented in Figure 5. The figure illustrates that the user has full control over the various options, e.g., add noise or not. 
The images shown in Figure 5 have uniform background radiance, calculated on the basis of the sky and sea temperatures only. Efforts are currently underway to introduce more realistic backgrounds ${ }^{53,54}$ in EOSTAR, which will result in improved synthetic sensor image. These images allow for the reliable representation of target contrast, e.g., to calculate detection probability and coverage diagrams.
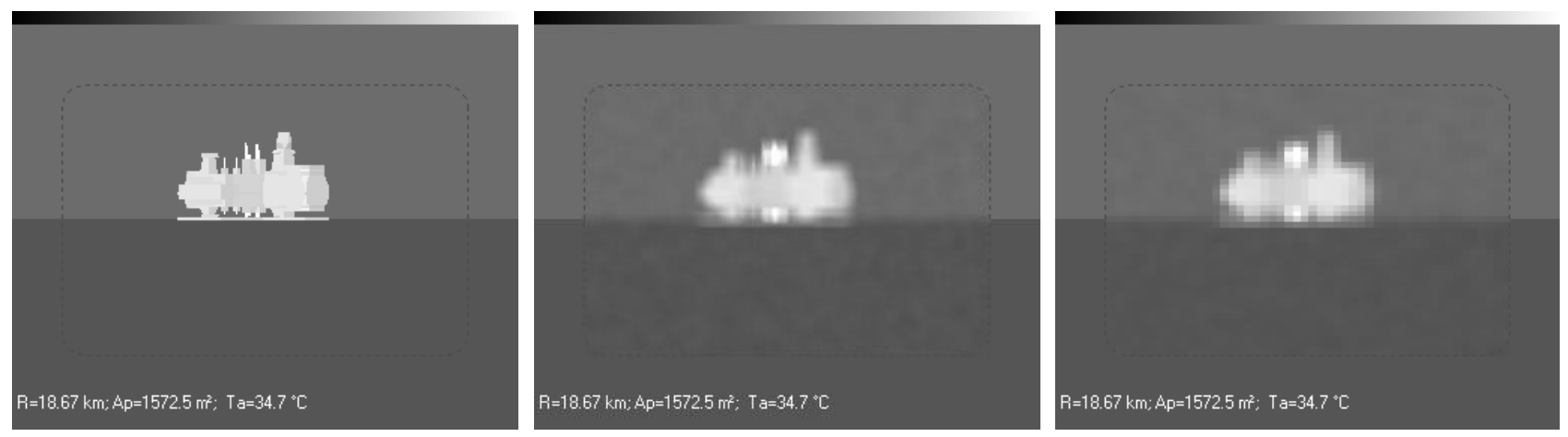

Figure 5: False color representation of a target after processing: refraction only (left), refraction, blur and noise (middle), and refraction, blur, noise and digitization. (Projected data in the bottom are not representative for the presented synthetic target.)

\section{CONCLUSION}

The performance of electro-optical sensors in long-range imaging applications is a complex function of the atmospheric conditions and the sensor system specifications. The choice of the optimal spectral band can be difficult. Moreover, atmospheric effects can both improve or degrade sensor performance. Depending on the conditions, the effective range can be reduced or extended to beyond the geometric horizon. Images may be largely distorted due to refractive effects and in some cases, multiple images of a single target are observed.

Based on standard meteorological data, EOSTAR allows studying atmospheric effects such as image deformation (refraction), turbulence effects (scintillation, blur and image motion), and transmission losses. The latter is spectrally resolved and split into contributions from aerosols, using the ANAM ${ }^{2,3,4}$ (Advanced Navy Aerosol Model), and molecules, using the external code MODTRAN ${ }^{1}$ (MODerate TRANsmission code). Path radiance is calculated from the transmission losses and the local air temperature along the ray trajectories, whereas the background radiance is currently computed from the sea and sky temperatures.

The June 2004 version of EOSTAR allows users to define their own targets as sets of segments. Each segment is assigned a number of physical and optical properties, which are used to calculate its spectral emission. This way a spectral signature of the overall target is obtained for a given heading and for a given atmospheric state. The spectral target signature is combined with the spectra of transmission and path radiance to simulate the spectral irradiance on the detector array. Image distortion such as refraction and blur are subsequently added using the vertical transfer function for the target range and the path-integrated values of $C_{\mathrm{n}}{ }^{2}$, respectively. Furthermore, noise is added and the image is digitized according to the sensor resolution.

EOSTAR is a PC-based computer program that assesses the performance of long-range infrared sensors in the marine atmosphere. EOSTAR is flexible (users can define their own sensors, targets and environments), completely mousecontrolled and instantaneously responding. Possible EOSTAR applications include mission planning, training and Monte-Carlo-type of simulations. A demo version and additional information is available from the EOSTAR website: http://www.tno.nl/instit/fel/os/fac/eostar.html. 


\section{REFERENCES}

1. A. Berk, G.P. Anderson, P.K. Acharya, J.H. Chetwynd, L.S. Bernstein, E.P. Shettle, M.W. Matthew and S.M. AdlerGolden, "Modtran4 user's manual", Air Force Research Laboratory, Space Vehicles Directorate - Air Force Material Command, Hanscom AFB, MA, 01731 - 3010 (1999).

2. S.G. Gathman, "Optical properties of the marine aerosol as predicted by the Navy aerosol model," Optical Engineering 22(1), 57-62 (1983).

3. S.G. Gathman, A.M.J. van Eijk and L.H. Cohen, "Characterizing large aerosols in the lowest level of the marine atmosphere," in Propagation and Imaging through the Atmosphere II, L.R. Bissonnette, Ed., Proc. SPIE Vol. 3433, pp. 41-52, Bellingham, Washington, USA (1998).

4. S.G. Gathman and M.H. Smith, "On the nature of surf generated aerosol and their effect on electro-optical systems," in Propagation and imaging through the atmosphere, L.R. Bissonnette and C. Dainty, Eds., Proc. SPIE Vol. 3125 , pp. 2-13, Bellingham, Washington, USA (1997).

5. J.M. Pernter and F.M. Exner, "Meteorologische Optik," Wilhelm Braumüller, Univesitäts-Verlagsbuchhandlung, Wien/Leipzig (1922).

6. R. Meyer, "Die Entstehung optischer Bilder durch Brechung und Spiegelung in der Atmosphäre," Meteorologische Zeitschrift 52, 405-408 (1935).

7. W.H. Lehn, "A simple parabolic model for the optics of the atmospheric surface layer," Applied Mathematical Modeling 9(12), 447-453 (1985).

8. S.Y. van der Werf, "Ray tracing and refraction in the modified US1976 atmosphere," Applied Optics 42(3), 354-366 (2003).

9. B. Edlén, "The dispersion of standard air," Journal of the Optical Society of America 43(5), 339-43 (1953).

10. B. Edlén, "The refractive index of air," Metroligia 2(2), 71-80 (1966).

11. R.W. Fenn, S.A. Clough, W.O. Gallery, R.E. Good, F.X. Kneizyz, J.D. Mill, L.S. Rothman, E.P. Shettle and F.E. Volz, "Optical and infrared properties of the atmosphere," in Handbook of geophysics and the space environment, Chapter 18, A.S. Jursa, Ed., pp. 18.1-18.80, Air Force Geophysics Laboratory, Air Force Systems Command, USA (1985).

12. J.C. Owens, "Optical refractive index of air: dependence on pressure, temperature and composition," Applied Optics 6(1), 51-59 (1967).

13. R.E. Hufnagel, "Propagation through atmospheric turbulence," in The Infrared Handbook, Capter 6, W.L. Wolfe and G.J. Zissis, Eds., pp. 6.1-6.56, The Infrared Information and Analysis (IRIA) Center, Environmental Research Institute of Michigan, Ann Arbor, MI, USA (1978).

14. E.L. Andreas and B.J. Thompson, Eds., Selected papers on turbulence in a refractive medium, Milestone Series MS 25 SPIE, Optical Engineering Press, Bellingham, USA (1990).

15. R.B. Beland, "Propagation through atmospheric turbulence," in The Infrared and Electro-Optical Systems Handbook, Atmospheric Propagation of Radiation, Volume 2, Chapter 2, F.G. Smith, Ed., pp. 157-234, Infrared Information Analysis Center, Ann Arbor, Michagan, USA (1993).

16. S. Church, "Atmospheric mirage and distortion modeling for IR target injection simulations," in Targets and Backgrounds: Characterization and Representation II, J.D. Gonglewski and K. Stein, Eds., Proc. SPIE Vol. 2742, pp. 122-135, Bellingham, Washington, USA (1996).

17. J.S. Watson, M.R. Wellfare, D.B. Chenault, S.E. Talele, B.T. Blume, M. Richards and L. Prestwood, "Irma multisensor predictive signature model," in Targets and Backgrounds: Characterization and Representation III, W.R. Watkins and D. Clement, Eds., Proc. SPIE Vol. 3062, pp. 158-172, Bellingham, Washington, USA (1997).

18. L.J. Forand, D. Dion, M. Duffy, S. Gathman, K. Littfin, A. de Jong, G. de Leeuw and K.L. Davidson, "An extensive analysis of low-level IR transmission measurements taken over a $15 \mathrm{~km}$ path during EOPACE with IRBLEM," in Propagation and Imaging through the Atmosphere II, L.R. Bissonnette, Ed., Proc. SPIE Vol. 3433, pp. 90-101, Bellingham, Washington, USA (1998).

19. D.A. Vaitekunas and O.E. Lawrence, "Infrared Scene Capabilities of SHIPIR," in AeroSense Conference, W.R. Watkins, D. Clement and W.R. Reynolds, Eds., Proc. SPIE Vol. 3699, pp. 92-102, Bellingham, USA (1999).

20. J.L. Forand, D. Dion, L. Gardenal and J. Gilbert, "Propagation resources in maritime environments (PRIME); an integrated model for the maritime battlespace," in Battlespace Atmopsheric and Clouds Impacts on Military Operations (BACIMO), CIRA, pp. 1-14, Colorado, USA (2000).

21. D. Dion, L. Gardenal, P. Lahaie and L. Forand, "Calculation and simulation of atmospheric refraction effects in maritime environments," in Atmospheric Propagation, Adaptive Systems and Laser Radar Technology for Remote 
Sensing, J.D. Gonglewski, G. Kamerman, A. Kohnle, U. Schreiber and C. Werner, Eds., Proc. SPIE Vol. 4167, pp. 1-9, Bellingham, Washington, USA (2001).

22. G.J. Kunz, M.M. Moerman and A.M.J. van Eijk, "ARTEAM: Advanced ray tracing with Earth atmospheric models," in AeroSense 2002, Targets and Background VIII: Characterization and Representation, W.R. Watkins, D. Clement and W.R. Reynolds, Eds., Proc. SPIE Vol. 4718, pp. 397-404, Bellingham, Washington, USA (2002).

23. G.J. Kunz, M.M. Moerman, A.M.J. van Eijk, S.M. Doss-Hammel and D. Tsintikidis, "EOSTAR: an electro-optical sensor performance model for predicting atmospheric refraction, turbulence, and transmission in the marine surface layer," in Optics in Atmospheric Propagation and Adaptive Systems VI, J.D. Gonglewski and K. Stein, Eds., Proc. SPIE Vol. 5237, pp. 81-92, Bellingham, Washington, USA (2003).

24. S.M. Doss-Hammel, D. Tsintikidis, A.M.J. van Eijk and G.J. Kunz, "Prediction and extrapolation: the use of the EOSTAR model in the marine infrared propagation environment," in Battlespace Atmopsheric and Clouds Impacts on Military Operations (BACIMO), CIRA, pp. 1-15, Colorado, USA (2003).

25. S. Doss-Hammel, D. Tsintikidis, A. van Eijk, and G. Kunz, "Refractive effects, turbulence and the EOSTAR model," in AeroSense 2004, Defence and Security, B.F. Andresen and G.F. Fulop, Eds., Proc. SPIE Vol. 5406, pp. 110, Bellingham, Washington, USA (2004).

26. R.B. Stull, "An introduction to boundary layer meteorology," Kluwer Academic Publishers, Dordrecht (1988).

27. S.P. Arya, "Introduction to micrometeorology," Academic Press, Inc., San Diego (1988).

28. D.A. Haugen, Ed., "Workshop on micrometeorology," American Meteorological Society, Boston (1973).

29. H.A. Panofsky and J.A. Dutton, "Atmospheric turbulence," John Wiley \& Sons, New York (1984).

30. K.L. Davidson, G.E. Schacher, C.W. Fairall and A.K. Goroch, "Verification of the bulk method for calculating overwater optical turbulence," Applied Optics 20(17), 498-502 (1981).

31. S.D. Smith, "Coefficients for sea surface wind stress, heat flux, and wind profiles as a function of wind speed and temperature," Journal of Geophysical Research 93(C12), 15,467-15,472 (1988).

32. W.T. Liu, K.B. Katsaros and J.A. Businger, "Bulk parameterization of air-sea exchanges of heat and water vapor including the molecular constraint at the interface," Journal of the Atmospheric Sciences 36(9), 1722-1735 (1979).

33. J. Kondo, "Air-sea bulk transfer coefficients in diabetic conditions," Boundary-Layer Meteorology 9, 91-112 (1975).

34. C.A. Paulson, "The mathematical representation of wind speed and temperature profiles in the unstable atmospheric surface layer," Journal of Applied Meteorology 9(12), 857-861 (1970).

35. V.E. Zuev, "Laser beams in the atmosphere," Consultants Bureau, New York, USA (1982).

36. C.A. Friehe, J.C. LaRue, F.H. Champagne, C.H. Gibson and G.F. Dreyer, "Effects of temperature and humidity fluctuations on the optical refractive index in the marine boundary layer," Journal of the Optical Society of America 65(12), 1502-1511 (1975).

37. G.J. Kunz, "A bulk model to predict optical turbulence in the marine surface layer", TNO-FEL Report Nr. FEL-96A053, TNO Physics and Electronics Laboratory, The Hague, The Netherlands (1996).

38. K.L. Davidson, T.M. Houlihan, C.W. Fairall and G.E. Schacher, "Observation of the temperature structure function parameter, $\mathrm{C}_{\mathrm{T}}{ }^{2}$, over the ocean," Boundary-Layer Meteorology 15, 507-523 (1978).

39. C.W. Fairall and S.E. Larsen, "Inertial-dissipation methods and turbulent fluxes at the air-ocean interface," Boundary-Layer Meteorology 34, 287-301 (1986).

40. J.C. Wyngaard and M.A. LeMone, "Behavior of the refractive index structure parameter in the entraining convective boundary layer," Journal of the Atmospheric Sciences 37(7), 1573-1585 (1980).

41. K.E. Kunkel and D.L. Walters, "Modeling the diurnal dependence of the optical refractive index structure parameter," Journal of Geophysical Research 88, 10.999-11.004 (1983).

42. W. Kohsiek, "Measuring $\mathrm{C}_{\mathrm{T}}{ }^{2}, \mathrm{C}_{\mathrm{Q}}{ }^{2}$ and $\mathrm{C}_{\mathrm{TQ}}$ in the unstable surface layer, and relations to the vertical fluxes of heat and moisture," Boundary-Layer Meteorology 24, 89-107 (1982).

43. W. Kohsiek, "Optical and in situ measuring of structure parameters relevant to temperature and humidity, and their application to the measureing of sensible and latent heat flux", NOAA Technical Memorandum, ERL WPL-96, Boulder, Co, USA (1982).

44. V. Thiermann and A. Kohnle, "Modelling of optically and IR effective atmospheric turbulence," in Atmospheric Propagation in the UV, Visible, IR and MM-Wave Region and Related Systems Aspects, AGARD-CP-454, pp. 19.119.12, Neuilly sur Seine, France (1989).

45. R.J. Hill, G.R. Ochs and J.J. Wilson, "Measuring surface-layer fluxes of heat and momentum using optical scintillation," Boundary-Layer Meteorology 58, 391-408 (1992).

46. R.J. Hill and G.R. Ochs, "Surface-layer similarity of the temperature structure parameter," Journal of the Atmospheric Sciences 49(15), 1348-1353 (1992). 
47. Y.A. Kravtsov and Y.I. Orlov, "Geometrical optics of inhomogeneous media," Springer, Berlin, Heidelberg, New York (1990).

48. W.G. Rees, C.M. Roach and C.H.F. Glover, "Inversion of atmospheric refraction data," Journal of the Optical Society of America 8(2), 330-338 (1991).

49. A.N. de Jong, "Refraction effects of atmospheric inhomogeneities along the path," in Optics in Atmospheric Propagation and Adaptive Systems VI, J.D. Gonglewski and K. Stein, Eds., Proc. SPIE Vol. 5237, pp. 105-116, Bellingham, Washington, USA (2003).

50. S. Hammel and N. Platt, "Topological description of mirage effects," in Vision Geometry IV, R.A. Melter, A.Y. Wu, F.L. Bookstein and W.D. Green, Eds., Proc. SPIE Vol 2573, pp. 398-406, Bellingham, Washington, USA (1995).

51. S. Doss-Hammel, "A passive ranging technique for objects within the marine surface layer", Biennial Review 2001, SPAWAR Systems Center, San Diego, USA (2001).

52. S. Doss-Hammel, A. Barrios and C. Zeisse, "The refractive amplification factor for infrared propagation in the marine atmospheric surface layer," in Battlespace Atmospheric and Clouds Impacts on Military Operations (BACIMO), CIRA, pp. 1-16, Colorado, USA (2001).

53. P. Schwering, "Maritime infrared background clutter," in Targets and Backgrounds: Characterization and Representation II, W.R. Watkins and D. Clement, Eds., Proc. SPIE Vol. 2742, pp. 255-266, Bellingham, Washington, USA (1996).

54. K.T. Constantikes and A.H. Zysnarski, "Computational models for synthetic marine infrared clutter," in Targets and Backgrounds: Characterization and Representation II, W.R. Watkins and D. Clement, Eds., Proc. SPIE Vol. 2742, pp. 154-168, Bellingham, Washington, USA (1996). 